\title{
Valoración de las competencias digitales en docentes universitarios
}

\author{
Value of digital competences in university professors \\ Jamine Pozú-Franco ${ }^{1}$, Fiorela Anaí Fernández-Otoya ${ }^{2}$, Liliana Muñoz- Guevara ${ }^{1}$

\section{RESUMEN}

El objetivo fue determinar el valor de las competencias digitales en 32 profesionales que ejercen docencia en una universidad privada de Lima. La metodología utilizada fue descriptiva y de carácter cuantitativo. Los datos fueron obtenidos mediante el Cuestionario Competencias Digitales, el cual se estructuró en cinco factores: alfabetización tecnológica, acceso y uso de la información, comunicación y colaboración, ciudadanía digital y creatividad e innovación. Los resultados revelaron que más de la mitad de los docentes valoraron como importantes o muy importantes todas las competencias evaluadas. Además 28 de las 43 competencias evaluadas fueron consideradas como muy importantes por la mitad o más de los docentes. Se concluye que la educación superior enfrenta como uno de sus más importantes desafíos la incorporación de los recursos tecnológicos al proceso E-A y la preparación de los docentes en el uso de dichos recursos.

PALABRAS CLAVE: competencias digitales, alfabetización digital, tecnología educacional, TIC, enseñanza superior

\section{SUMMARY}

The objective was to determine the value of digital skills in 32 professionals who teach in a private university in Lima. The methodology used was descriptive and quantitative in nature. The data were obtained through the Digital Competences Questionnaire, which was structured in five factors: technological literacy, access and use of information, communication and collaboration, digital citizenship, and creativity and innovation. The main results revealed that more than half of participants valued as important or very important all of the competences assessed. Furthermore, 28 out from the 43 competences assessed were valued as very important by half or more than half of participants. It is concluded that higher education has as one of its more important challenges incorporating technological resources in the teaching-learning process and training of faculty in the use of that technology.

KEY WORDS: digital skills, digital literacy, educational technology, ICT, higher education

\section{INTRODUCCIÓN}

El creciente adelanto tecnológico viene originando trascendentales cambios en todos los sectores sociales, concretamente, en el sector educativo; donde se ha planteado la necesidad de incorporar la tecnología en la práctica pedagógica. Por ello, es esencial que todos los docentes posean competencias tecnológicas indispensables para incorporar sus conocimientos y habilidades en el proceso Enseñanza Aprendizaje (PEA).

Usar la tecnología es una práctica diaria en los profesionales que ejercen la docencia universitaria dado que los recursos y materiales digitales permiten complementar su metodología de enseñanza. Este escenario destaca la notabilidad de las competencias digitales en la educación (Marciales et al., 2015). No

Universidad Peruana Cayetano Heredia. Lima, Perú.

2 Universidad Católica Santo Toribio de Mogrovejo. Lambayeque, Perú. 
obstante, existe una brecha entre las herramientas tecnológicas disponibles en las aulas y el uso educativo que los docentes puedan darles (Instefjord, 2014). Esto, como consecuencia de la ausencia de estímulos adecuados para su uso en la enseñanza, falta de evidencia basada en la investigación para informar la manera de incorporarla en el PEA (Organización para la Cooperación y Desarrollo Económicos [OCDE], 2008) o a causa de la brecha digital (Montero et al., 2019).

Los desafíos del siglo XXI plantearon la necesidad de un nuevo enfoque en el PEA, cimentado en los principios de excelencia, calidad y pertinencia (Ander-Egg, 2005). Es así que el sistema educativo universitario acogió del mundo laboral el término competencia para referirse a la cualidad del docente universitario y como elemento transversal para la formación de las personas. De allí que una educación basada en competencias responde a una educación de calidad, sustentada en la trasformación de los perfiles profesionales, que pretende brindar las mismas oportunidades para toda la sociedad (Tobón, 2016).

Dialogar sobre competencias en educación es remitirse a una perspectiva educativa transformadora. No obstante, muchas universidades forman estudiantes para una sociedad industrial, y no para la sociedad actual. Es así que existen referentes internacionales (Acuerdo de Bolonia, el Proyecto Tuning, entre otros) y experiencias universitarias donde se aplica con éxito el enfoque por competencias. Así, existen universidades que vienen implementando este enfoque cuyos resultados han sido favorables, conllevando un rediseño de los planes de estudio, en las metodologías y formas de evaluar.

En la formación de profesionales no existe un único concepto sobre competencia. Así, el Consejo y Parlamento Europeo, en el 2006, la definió como la unión de conocimientos, capacidades y actitudes contextualizados. Mientras que la Comisión Europea (2008) la define como la capacidad de emplear las consecuencias del aprendizaje en un contexto explícito. Además, señala que la competencia no se limita a componentes cognitivos (concepto, teoría, o conocimiento implícito), sino que también comprende elementos funcionales (destrezas técnicas), propiedades interpersonales (destrezas sociales y de organización) y valores moralistas; es decir, una competencia se compone de conocimientos, habilidades y actitudes.
La competencia es una facultad del ser humano para combinar una serie de capacidades y alcanzar una meta específica en un contexto concreto, procediendo de forma oportuna y con significado moralista (Ministerio de Educación [MINEDU], 2016). En ese sentido, alguien es competente cuando comprende la situación a afrontar; evalúa los medios (conocimientos y habilidades) que tiene a su disposición para resolverla, seleccionando los más pertinentes a la situación, para luego tomar decisiones y ejecutarlas; de allí el dicho: una competencia se manifiesta en la acción. Las competencias pueden ser adquiridas, transmitidas, aprendidas, enseñadas y desarrolladas a lo largo de toda la vida. Su adquisición dependerá, en gran medida, del esfuerzo de cada persona.

El Consejo y Parlamento Europeo, durante el año 2006, adoptó por primera vez un antecedente que define e identifica las competencias clave para la formación permanente, realización personal, ciudadanía activa e inclusión social (Comunidades Europeas, 2007). Estas competencias fueron: Comunicación en la lengua materna, Comunicación en lenguas extranjeras, Competencia matemática y competencias básicas en ciencia y tecnología, Competencia digital, Aprender a aprender, Competencias sociales y cívicas, Sentido de la iniciativa y espíritu de empresa y Conciencia y expresión culturales.

Es así que, desde el 2007, la competencia digital es considerada como una de las ocho competencias clave para el aprendizaje permanente, trabajo, ocio y comunicación. Ser competente digitalmente consiste en usar los ordenadores para adquirir, valorar, acopiar, crear, exponer e intercambiar información para informar e interactuar en redes colaborativas. Esto supone usar de manera segura y crítica las tecnologías, siendo indispensable conocer y comprender el carácter, función y oportunidades que ofrece la tecnología en escenarios habituales de la vida personal, profesional y social (Comunidades Europeas, 2007).

Diversos autores refieren que un buen docente universitario se identifica por ser competente digitalmente (Zabalza, 2007); es decir, por dominar las tecnologías de información y comunicación (TIC) en diferentes contextos, saber cómo utilizarlas y apropiarse de ellas (Ferrari, 2012). Dominar una herramienta requiere conocerla para posteriormente usarla. En este proceso de aprendizaje se investigan diferentes aspectos como conocer cómo funciona, considerando la posibilidad de la ayuda de otros que están más familiarizados con ella. 
Ante ello, surge la necesidad de investigar cómo valoran los docentes universitarios los diversos factores de la competencia digital, cuál es el factor más valorado y qué desempeño es mejor valorado en cada factor. Los factores a ser considerados son los cinco propuestos por Mengual y Roig (2012): alfabetización tecnológica, acceso y uso de la información, comunicación y colaboración, ciudadanía digital y creatividad e innovación. Por tanto, este estudio tuvo como objetivo determinar la valoración que tienen los docentes universitarios de una universidad de Lima sobre las competencias digitales a partir de factores que reconocen el uso de tecnologías en un entorno universitario y de cómo se apropian de ellas para aplicarlas como herramientas pedagógicas.

\section{MÉTODOS}

La población estuvo conformada por 48 profesionales que ejercen docencia en una universidad particular de Lima en la modalidad presencial, semipresencial y virtual. La muestra de 32 docentes universitarios, de ambos sexos, fue seleccionada de manera no aleatoria, por conveniencia. Se consideraron como criterios de inclusión que todos los docentes trabajen en la universidad seleccionada, que tengan una computadora o laptop y que acepten libremente participar en el estudio (consentimiento informado).

Para recoger la información de los docentes, se empleó el cuestionario Competencias Digitales, adaptado de Mengual (2011), que evalúa el nivel de importancia que los docentes universitarios le asignan a las competencias indicadas en los 43 ítems, cuyas respuestas fueron valoradas mediante una escala tipo Likert con las alternativas: nada importante, poco importante, más o menos importante, importante y muy importante. El cuestionario estuvo estructurado como se explica a continuación:

Título y presentación del instrumento.

1. Datos personales respecto al uso de las TIC. Se elaboraron siete preguntas que permiten definir la muestra (disposición de computadora y acceso a internet, número de horas de uso del ordenador, formación en el uso y manejo del ordenador, cantidad de horas recibidas de capacitación sobre el uso de las TIC, total de años utilizando computadoras, el uso que se le da a la tecnología con miras a contribuir en el perfeccionamiento de la calidad profesional y el grado de integración de las TIC en la asignatura que enseña).
2. Batería de ítems estructurados en cinco factores: F1: Alfabetización tecnológica. Consta de 7 ítems que expresan la valoración sobre los conceptos que se posee respecto al uso que se le da al ordenador con sus respectivas aplicaciones en el contexto laboral.

F2: Acceso y uso de la información. Posee 8 ítems que se refieren a cómo buscar información selecta, valorando las diferentes fuentes, su origen y respetando la autoría.

F3: Comunicación y colaboración. Consta de 8 ítems que permiten valorar la forma como se comunican los docentes en los diferentes entornos digitales, comparten sus recursos online y se conectan, colaboran, interactúan y participan con otros por medio de herramientas digitales.

F4: Ciudadanía digital. Consta de 8 ítems que expresan la valoración docente respecto a asumir un compromiso ético y personal para la utilización legal, segura y responsable de la información digital y las herramientas TIC en la comunicación y colaboración digital para el aprendizaje continuo. F5: Creatividad e innovación. Consta de 12 ítems que expresan la valoración sobre la incorporación de las sapiencias en TIC para la práctica pedagógica.

\section{Cierre y agradecimiento.}

Se inició la investigación propiciando el contacto con las autoridades de la universidad en la que se efectuaría el estudio, a fin de solicitar la autorización correspondiente. Una vez aceptada la solicitud, se seleccionó la muestra mediante el método no probabilístico por conveniencia. Los participantes seleccionados recibieron una explicación sobre el estudio y procedieron a firmar el consentimiento informado; asimismo, realizaron las coordinaciones de fecha y lugar para aplicar el cuestionario de Competencias Digitales. Las respuestas fueron anónimas. A los participantes se les indicó verbalmente las instrucciones recalcándoles la importancia de una repuesta veraz. Los datos fueron recolectados individualmente y durante 01 mes durante el año 2017.

Para procesar los datos se recurrió al uso del SPSS 25. Para medir nivel de importancia de las competencias digitales de los docentes universitarios con un total de 43 ítems, se aplicó el criterio de confiabilidad del instrumento por consistencia interna, mediante el alfa de Cronbach, arrojando un valor de .975 , por lo que se considera que las puntuaciones obtenidas con el cuestionario son muy confiables. 
Los cinco factores también obtuvieron alfas elevados: Alfabetización tecnológica $(0,884)$, Acceso y uso de la información $(0,925)$, Comunicación y colaboración $(0,912)$, Ciudadanía digital $(0,959)$ y Creatividad e innovación $(0,961)$.

\section{RESULTADOS}

Los resultados del presente estudio son presentados considerando los cinco factores del Cuestionario Competencias Digitales propuesto por Mengual (2011).

Tabla 1.

Porcentaje de Participantes con Diversos Niveles de Valoración de la Alfabetización Tecnológica

\begin{tabular}{|c|c|c|c|c|c|}
\hline Factor 1: alfabetización tecnológica & $\begin{array}{l}\text { Nada } \\
\text { Importante }\end{array}$ & $\begin{array}{l}\text { Poco } \\
\text { Importante }\end{array}$ & $\begin{array}{l}\text { Más o } \\
\text { menos } \\
\text { importante }\end{array}$ & Importante & $\begin{array}{l}\text { Muy } \\
\text { Importante }\end{array}$ \\
\hline $\begin{array}{l}\text { Manejar los recursos de una computadora a } \\
\text { través de los distintos Sistemas Operativos } \\
\text { (Windows, Linux, Mac). }\end{array}$ & $0,00 \%$ & $0,00 \%$ & $3,13 \%$ & $59,38 \%$ & $37,50 \%$ \\
\hline $\begin{array}{l}\text { Utilizar herramientas ofimáticas para el } \\
\text { tratamiento de la información (editores de } \\
\text { texto, editores de presentaciones en formato } \\
\text { digital, hojas de cálculo, bases de datos, } \\
\text { etc.). }\end{array}$ & $0,00 \%$ & $0,00 \%$ & $3,13 \%$ & $40,63 \%$ & $56,25 \%$ \\
\hline $\begin{array}{l}\text { Dominar herramientas de tratamiento } \\
\text { de imagen, audio y video digital (Gimp, } \\
\text { PhotoShop, Audacity, Cdex, Moviemaker, } \\
\text { etc.). }\end{array}$ & $3,13 \%$ & $6,25 \%$ & $21,88 \%$ & $46,88 \%$ & $21,88 \%$ \\
\hline $\begin{array}{l}\text { Usar herramientas digitales existentes } \\
\text { y emergentes de forma efectiva para la } \\
\text { localización, el análisis, y la evaluación de } \\
\text { recursos de información. }\end{array}$ & $0,00 \%$ & $3,13 \%$ & $12,50 \%$ & $53,13 \%$ & $31,25 \%$ \\
\hline $\begin{array}{l}\text { Utilizar herramientas de comunicación } \\
\text { basadas en servicios de correo electrónico } \\
\text { de tipo cliente y webmail (Gmail, Outlook, } \\
\text { Eudora, Thunderbird, etc.). }\end{array}$ & $0,00 \%$ & $0,00 \%$ & $3,13 \%$ & $28,13 \%$ & $68,75 \%$ \\
\hline $\begin{array}{l}\text { Desarrollar conversaciones online a través } \\
\text { de herramientas de comunicación síncrona } \\
\text { vía Web (chat, servicios de mensajería } \\
\text { instantánea, Skype, herramientas de } \\
\text { videoconferencia, etc.). }\end{array}$ & $0,00 \%$ & $0,00 \%$ & $6,25 \%$ & $46,88 \%$ & $46,88 \%$ \\
\hline $\begin{array}{l}\text { Desarrollar conversaciones online a } \\
\text { través de herramientas de comunicación } \\
\text { asíncrona vía Web, tanto tradicionales como } \\
\text { emergentes (foros, listas de distribución, } \\
\text { grupos de discusión, tweets, etc.). }\end{array}$ & $0,00 \%$ & $0,00 \%$ & $6,25 \%$ & $50,00 \%$ & $43,75 \%$ \\
\hline $\begin{array}{l}\text { Efectuar trabajos colaborativos a través } \\
\text { de herramientas online de tipo Groupware } \\
\text { (GoogleDocs, Kolab, etc.) }\end{array}$ & $0,00 \%$ & $6,25 \%$ & $12,50 \%$ & $31,25 \%$ & $50,00 \%$ \\
\hline $\begin{array}{l}\text { Dominar herramientas web para compartir } \\
\text { y publicar recursos en línea (GoogleVideo, } \\
\text { Youtube, Flickr, Slideshare, Scribd, etc.). }\end{array}$ & $0,00 \%$ & $6,25 \%$ & $3,13 \%$ & $50,00 \%$ & $40,63 \%$ \\
\hline $\begin{array}{l}\text { Usar de forma efectiva plataformas de } \\
\text { e-learning/b-learning para la formación } \\
\text { y colaboración online (Moodle, Dokeos, } \\
\text { WebCt, Chamilo, Blackboard, etc.). }\end{array}$ & $0,00 \%$ & $9,38 \%$ & $3,13 \%$ & $18,75 \%$ & $68,75 \%$ \\
\hline
\end{tabular}


En la Tabla 1, se muestra la valoración de los docentes respecto al Factor 1, Alfabetización Tecnológica. Los porcentajes más altos se sitúan en las valoraciones muy importante e importante. Así 68,75\% refieren que es muy importante usar herramientas de comunicación apoyadas en el correo electrónico (Gmail, Outlook, Eudora, Thunderbird, etc.); así como, utilizar las plataformas e-learning/blearning para formar y colaborar online (Moodle, Dokeos, WebCt, Chamilo, Blackboard).

Además, existe un considerable grupo de docentes que refieren que es importante saber utilizar los recursos de un ordenador mediante los diferentes Sistemas Operativos; dominar herramientas para el tratamiento de imagen, audio y video digital; usar herramientas digitales existentes y emergentes de forma efectiva para localizar, analizar y evaluar recursos de información; desarrollar pláticas online usando herramientas de comunicación asincrónica disponibles en la Web y dominar herramientas web para compartir y publicar recursos online.

La Tabla 2 exhibe la valoración docente en relación con el Factor de Acceso y Uso de la Información, centrándose los porcentajes más altos en la valoración "importante", específicamente en el ítem definición de problemas a solucionar usando las TIC; en los ítems recupera, organiza y gestiona la información mediante herramientas y servicios tecnológicos, y demuestra el beneficio del conocimiento adquirido para tomar decisiones al momento de resolver un problema, manifestaron también la valoración "importante", el $56,25 \%$ y $50 \%$ de los participantes, respectivamente.

Tabla 2.

Porcentaje de Participantes con Diversos Niveles de Valoración del Acceso y Uso de la Información

\begin{tabular}{|c|c|c|c|c|c|}
\hline $\begin{array}{l}\text { Factor 2: Acceso y uso de la } \\
\text { información }\end{array}$ & $\begin{array}{l}\text { Nada } \\
\text { Importante }\end{array}$ & $\begin{array}{l}\text { Poco } \\
\text { Importante }\end{array}$ & $\begin{array}{l}\text { Más o } \\
\text { menos } \\
\text { importante }\end{array}$ & Importante & $\begin{array}{l}\text { Muy } \\
\text { Importante }\end{array}$ \\
\hline $\begin{array}{l}\text { Definir problemas a resolver con el } \\
\text { uso de las TIC. }\end{array}$ & $0,00 \%$ & $9,38 \%$ & $6,25 \%$ & $56,25 \%$ & $28,13 \%$ \\
\hline $\begin{array}{l}\text { Diseñar un proyecto de investigación } \\
\text { sobre la base de un problema a } \\
\text { resolver, identificando los recursos } \\
\text { TIC más adecuados. }\end{array}$ & $0,00 \%$ & $3,13 \%$ & $15,63 \%$ & $46,88 \%$ & $34,38 \%$ \\
\hline $\begin{array}{l}\text { Planificar búsquedas de información } \\
\text { para la resolución de problemas. }\end{array}$ & $0,00 \%$ & $6,25 \%$ & $6,25 \%$ & $43,75 \%$ & $43,75 \%$ \\
\hline $\begin{array}{l}\text { Efectuar la recuperación, organización } \\
\text { y gestión de la información utilizando } \\
\text { herramientas y servicios tecnológicos. }\end{array}$ & $0,00 \%$ & $3,13 \%$ & $12,50 \%$ & $50,00 \%$ & $34,38 \%$ \\
\hline $\begin{array}{l}\text { Identificar la información relevante } \\
\text { evaluando las distintas fuentes y su } \\
\text { procedencia. }\end{array}$ & $0,00 \%$ & $6,25 \%$ & $6,25 \%$ & $37,50 \%$ & $50,00 \%$ \\
\hline $\begin{array}{l}\text { Sintetizar la información seleccionada } \\
\text { organizándola adecuadamente para la } \\
\text { construcción y asimilación del nuevo } \\
\text { conocimiento. }\end{array}$ & $0,00 \%$ & $0,00 \%$ & $6,25 \%$ & $37,50 \%$ & $53,13 \%$ \\
\hline $\begin{array}{l}\text { Demostrar la utilidad del } \\
\text { conocimiento obtenido para la toma } \\
\text { de decisiones en la solución de un } \\
\text { problema. }\end{array}$ & $0,00 \%$ & $0,00 \%$ & $6,25 \%$ & $50,00 \%$ & $43,75 \%$ \\
\hline $\begin{array}{l}\text { Devolver a la comunidad en términos } \\
\text { de recursos de información digitales, } \\
\text { la solución de un problema. }\end{array}$ & $0,00 \%$ & $3,13 \%$ & $9,38 \%$ & $43,75 \%$ & $43,75 \%$ \\
\hline
\end{tabular}


Tabla 3.

Porcentaje de Participantes con Diversos Niveles de Valoración de la Comunicación y Colaboración

\begin{tabular}{|c|c|c|c|c|c|}
\hline Factor 3: Comunicación y colaboración & $\begin{array}{l}\text { Nada } \\
\text { Importante }\end{array}$ & $\begin{array}{l}\text { Poco } \\
\text { Importante }\end{array}$ & $\begin{array}{l}\text { Más o } \\
\text { menos } \\
\text { importante }\end{array}$ & Importante & $\begin{array}{l}\text { Muy } \\
\text { Importante }\end{array}$ \\
\hline $\begin{array}{l}\text { Compartir entornos y medios digitales para } \\
\text { la colaboración y publicación de recursos } \\
\text { electrónicos con los compañeros. }\end{array}$ & $0,00 \%$ & $3,13 \%$ & $6,25 \%$ & $37,50 \%$ & $53,13 \%$ \\
\hline $\begin{array}{l}\text { Interactuar con expertos u otras personas } \\
\text { empleando redes sociales y canales de } \\
\text { comunicación basados en TIC. }\end{array}$ & $0,00 \%$ & $0,00 \%$ & $3,13 \%$ & $34,38 \%$ & $62,50 \%$ \\
\hline $\begin{array}{l}\text { Comunicar efectivamente información e } \\
\text { ideas a múltiples audiencias, usando una } \\
\text { variedad de medios, formatos y plataformas. }\end{array}$ & $0,00 \%$ & $0,00 \%$ & $9,38 \%$ & $31,25 \%$ & $59,38 \%$ \\
\hline $\begin{array}{l}\text { Desarrollar una comprensión cultural y una } \\
\text { conciencia global mediante la vinculación } \\
\text { con profesionales de otras culturas. }\end{array}$ & $0,00 \%$ & $6,25 \%$ & $12,50 \%$ & $37,50 \%$ & $43,75 \%$ \\
\hline $\begin{array}{l}\text { Comunicarse con expertos de otras áreas a } \\
\text { través de canales de comunicación basados } \\
\text { en TIC. }\end{array}$ & $0,00 \%$ & $3,13 \%$ & $9,38 \%$ & $34,38 \%$ & $53,13 \%$ \\
\hline $\begin{array}{l}\text { Formar equipos de trabajo inter y } \\
\text { multidisciplinar para el desarrollo de } \\
\text { proyectos o la resolución de problemas. }\end{array}$ & $0,00 \%$ & $6,25 \%$ & $6,25 \%$ & $31,25 \%$ & $56,25 \%$ \\
\hline $\begin{array}{l}\text { Crear y dinamizar redes y comunidades } \\
\text { profesionales del conocimiento para el } \\
\text { trabajo colaborativo en entornos virtuales. }\end{array}$ & $0,00 \%$ & $0,00 \%$ & $9,38 \%$ & $34,38 \%$ & $56,25 \%$ \\
\hline Compartir experiencias en redes sociales. & $0.00 \%$ & $0,00 \%$ & $3,13 \%$ & $53,13 \%$ & $12,50 \%$ \\
\hline
\end{tabular}

Seguidos de un considerable porcentaje de docentes que valora como muy importante a los ítems resumir información selecta organizándola apropiadamente para construir y asimilar el nuevo conocimiento, y el ítem sobre identificar información selecta valorando las diferentes fuentes y su origen, con un $53,13 \%$ y $50 \%$ respectivamente.

La Tabla 3 permite apreciar la valoración del Factor 3, Comunicación y Colaboración, en el que se destaca como muy importante el hecho de interactuar con personas explotando las redes sociales y canales de comunicación $(62,50 \%)$; comunicar de manera efectiva información e ideas mediante variados medios electrónicos y plataformas (59.38\%); trabajar en equipo de manera multi e interdisciplinar con el propósito de resolver problemas o desarrollar proyectos $(56,25 \%)$; idear y fortalecer comunidades y redes profesionales para trabajar colaborativamente en entornos virtuales $(56,25 \%)$; tener comunicación con profesionales mediante canales de comunicación (53,13\%); compartir ambientes y medios digitales para colaborar y publicar recursos digitales con sus colegas $(53,13 \%)$, además, un $53,13 \%$ valoraron como importante el hecho de compartir experiencias en redes sociales.

En la Tabla 4 se expone la valoración del Factor de Ciudadanía Digital por parte de los docentes, quienes resaltaron su importancia en todos los ítems que lo conforman. Así, en el ítem fomenta el uso responsable, legal y seguro de las TIC e información; un $78,13 \%$ señaló que es muy importante; de igual forma, el 71,88\% revela la importancia de asumir un compromiso ético al usar la información digital y las TIC, envolviendo los derechos de autoría, propiedad intelectual y documentación apropiada de las fuentes; $62,50 \%$ demostraron la importancia de asumir compromisos para aprender durante toda su vida usando las TIC; un 59.38\% mostraron la importancia de 
Tabla 4.

Porcentaje de Participantes con Diversos Niveles de Valoración de la Ciudadanía Digital

\begin{tabular}{llll}
\hline Factor 4: Ciudadanía digital & $\begin{array}{l}\text { Nada } \\
\text { Importante }\end{array}$ & $\begin{array}{l}\text { Poco } \\
\text { Importante }\end{array}$ & $\begin{array}{l}\text { Más o } \\
\text { menos } \\
\text { importante }\end{array}$
\end{tabular}$\quad$\begin{tabular}{l} 
Importante \\
Importante \\
\hline
\end{tabular}

Asumir un compromiso ético en el uso de la información digital y de las TIC, incluyendo el respeto por los derechos de autor, la propiedad intelectual y la documentación adecuada de las fuentes.

Promover el uso seguro, legal y responsable de la información y de las TIC.

Mostrar una actitud positiva frente al uso de las TIC apoyando la colaboración, el aprendizaje y la productividad.

Demostrar responsabilidad personal para el aprendizaje a lo largo de la vida utilizando las TIC.

Ejercer liderazgo para la ciudadanía digital.

Utilizar de forma equitativa herramientas y recursos digitales apropiados.

Comprender la etiqueta digital (netiqueta)

desarrollando interacciones sociales responsables relacionadas con uso de la información y las TIC.

Desarrollar una comprensión de culturas y conciencia global relacionándose con profesionales de otras culturas, mediante el uso de herramientas de comunicación y colaboración de la era digital.

$\begin{array}{lllll}0,00 \% & 3,13 \% & 6,25 \% & 18,75 \% & 71,88 \%\end{array}$

$\begin{array}{lllll}0,00 \% & 0,00 \% & 3,13 \% & 18,75 \% & 78,13 \%\end{array}$

$\begin{array}{lllll}0,00 \% & 0,00 \% & 6,25 \% & 34,38 \% & 59,38 \%\end{array}$

$\begin{array}{lllll}0,00 \% & 3,13 \% & 3,13 \% & 31,25 \% & 62,50 \%\end{array}$

$\begin{array}{lllll}3,13 \% & 3,13 \% & 9,38 \% & 34,38 \% & 50,00 \%\end{array}$

$3,13 \% \quad 0,00 \% \quad 0,00 \% \quad 43,75 \% \quad 53,13 \%$

$\begin{array}{lllll}3,13 \% & 3,13 \% & 6,25 \% & 34,38 \% & 53,13 \%\end{array}$

$\begin{array}{lllll}3,13 \% & 3,13 \% & 3,13 \% & 43,75 \% & 46,88 \%\end{array}$

actuar positivamente frente al empleo de las TIC para aprender colaborativa y productivamente; un 53,13\% en los ítems usar equitativamente herramientas y recursos digitales apropiados; así como comprender la etiqueta digital (netiqueta) para el intercambio responsable de la información e interacción utilizando las TIC; 50\% en ejercer autoridad en la ciudadanía digital y 46.88\% en comprender las culturas y conciencia integral para interaccionar con profesionales mediante el empleo de herramientas que permiten comunicar y colaborar digitalmente.

En la Tabla 5 se exhiben los resultados correspondientes al Factor Creatividad e Innovación, cuyas valoraciones más altas se sitúan en el nivel muy importante en casi todos los ítems. Así, los ítems mejor valorados con un $68.75 \%$, como muy importante son los que se refieren a la adaptación a nuevas circunstancias y escenarios tecnológicos; el desarrollo de experiencias que buscan estimular la creatividad e innovación y la integración de recursos y herramientas digitales que promueven la capacidad para aprender y ser creativo. Además, el 59.38\% de docentes destaca como muy importante el hecho de emplear los conocimientos adquiridos para la generación de opiniones, productos o procesos utilizando las TIC y expandir la garantía de autoperfeccionamiento profesional en su contexto laboral utilizando las herramientas tecnológicas.

Así también, los ítems relacionados con la elaboración de trabajos originales como medios de expresión personal o grupal empleando las TIC, 
Tabla 5.

Porcentaje de Participantes con Diversos Niveles de Valoración de la Creatividad e Innovación

\begin{tabular}{llll}
\hline Factor 5: Creatividad e innovación & $\begin{array}{l}\text { Nada } \\
\text { Importante }\end{array}$ & $\begin{array}{l}\text { Poco } \\
\text { Importante }\end{array}$ & $\begin{array}{l}\text { Más o menos } \\
\text { importante }\end{array}$
\end{tabular} Importante $\begin{aligned} & \text { Muy } \\
& \text { Importante }\end{aligned}$

conocimientos en TIC en la práctica $\quad 0,00 \% \quad 3,13 \% \quad 6,25 \% \quad 40,63 \% \quad 50,00 \%$

profesional.

Adaptarse a nuevas situaciones y entornos tecnológicos.

$\begin{array}{lllll}0,00 \% & 0,00 \% & 6,25 \% & 25,00 \% & 68,75 \%\end{array}$

Utilizar el conocimiento existente para generar nuevas ideas, productos o procesos mediante las TIC.

$\begin{array}{lllll}0,00 \% & 0,00 \% & 3,13 \% & 37,50 \% & 59,38 \%\end{array}$

Crear trabajos originales como medios de expresión personal o grupal utilizando las TIC, como parte de su aprendizaje permanente y reflexivo.

$\begin{array}{lllll}0,00 \% & 0,00 \% & 9,38 \% & 37,50 \% & 53,13 \%\end{array}$

Usar modelos y simulaciones para explorar sistemas y temas complejos utilizando las TIC.

$\begin{array}{lllll}0,00 \% & 6,25 \% & 12,50 \% & 37,50 \% & 43,75 \%\end{array}$

Identificar tendencias previendo las posibilidades de utilización de las TIC.

$\begin{array}{lllll}0,00 \% & 9,38 \% & 3,13 \% & 46,88 \% & 40,63 \%\end{array}$

Usar múltiples procesos y diversas perspectivas para explorar soluciones alternativas al problema dado.

$3,13 \% \quad 3,13 \% \quad 0,00 \% \quad 46,88 \% \quad 43,75 \%$

Reconocer las condiciones y los contextos que exigen el empleo de las TIC (dónde, cuándo, cómo).

Participar en comunidades profesionales del conocimiento que empleen las TIC.

$0,00 \%$

$00 \%$

$6,25 \%$

$40,63 \%$

$53,13 \%$

Desarrollar experiencias que estimulen el pensamiento creativo e innovador.

$0,00 \%$

$0,00 \%$

$28,13 \%$

$68,75 \%$

Integrar herramientas y recursos digitales para promover la capacidad de aprendizaje y creatividad.

$\begin{array}{lllll}3,13 \% & 0,00 \% & 3,13 \% & 25,00 \% & 68,75 \%\end{array}$

Tender a la efectividad y autorrenovación profesional incorporando las TIC en su contexto laboral.

$\begin{array}{lllll}0,00 \% & 3,13 \% & 3,13 \% & 34,38 \% & 59,38 \%\end{array}$

como parte de su aprendizaje permanente y reflexivo y la participación en redes profesionales para generar conocimiento con empleo de las TIC, son valorados como muy importante por el 53.13\% de los participantes. Finalmente, los ítems que buscan valorar la incorporación de los conocimientos que se poseen sobre las TIC para aplicarlos en su quehacer pedagógico y saber reconocer las circunstancias y los contextos que demandan el uso de las herramientas tecnológicas (dónde, cuándo, cómo), fueron considerados por los docentes como muy importantes en un $50 \%$. 
Resumiendo los resultados, se aprecia que más de la mitad de los docentes valoraron como importantes o muy importantes todas las competencias evaluadas. Además 28 de las 43 competencias evaluadas fueron consideradas como muy importantes por la mitad o más de los docentes.

\section{DISCUSIÓN}

Los resultados exhiben el reconocimiento sustancial que los docentes otorgan a las competencias digitales en el quehacer pedagógico, las cuales son percibidas como un factor primordial para innovar, producir y competir. Entendiéndose a la competencia digital como una serie de conocimientos, destrezas y actitudes indispensables para usar las herramientas tecnológicas con el propósito de elaborar contenidos académicos, solucionar situaciones problemáticas, informar y gestionar, crear y compartir información que permite cimentar conocimiento (Ferrari, 2012).

Desde la perspectiva de las Comunidades Europeas (2007), el conocimiento tecnológico hace referencia a la noción que se posee de las principales herramientas informáticas (hojas de cálculo, procesadores de contenidos, discos duros virtuales y bases de datos) para comprender los posibles beneficios y peligros que brinda el uso indiscriminado de la Internet y los medios electrónicos en el desenvolvimiento profesional, entretenimiento, aprendizaje e investigación. Además, proponen como habilidades necesarias para usar herramientas que permitan generar, exhibir y comprender información, acentuando el acceso a servicios prestados en Internet: búsqueda, obtención y tratamiento de la información que fomente pensar de manera crítica, creativa e innovadora. Y manifiestan que aplicar las TIC demanda de un proceder reflexivo y crítico frente a la información aprovechable, así como usar responsablemente los recursos que propician la interactividad.

Ser competente digitalmente conlleva que los docentes usen de manera crítica y segura las TIC (Unión Europea, 2011); es decir, reflexionen sobre cómo, cuándo y para qué utilizarlas, así como trazar nuevas maneras de aprender, enseñar, tomar la decisión de apropiarse o no de ellas y utilizarlas de manera innovadora en la enseñanza, aprovechando de las preeminencias que ofrecen las herramientas tecnológicas (Flores-Lueg \& Roig, 2016). Para fines de este estudio, para evaluar el nivel de valoración de las competencias digitales por los docentes universitarios, se consideraron los cinco factores propuestos por
Mengual y Roig (2012): alfabetización tecnológica, acceso y uso de la información, comunicación y colaboración, ciudadanía digital y creatividad e innovación.

Referente a la alfabetización digital, se evidencia el reconocimiento por los docentes de la importancia del uso de herramientas para la comunicación, así como de las plataformas e-learning/b-learning en la formación y colaboración en línea y del uso de herramientas como videos, ppt, word y hojas de cálculo Excel que permiten la elaboración de materiales digitales. Estos resultados coinciden con las observaciones de Ocampo et al. (2015), quienes, al analizar la percepción docente acerca del proceso de certificación en modalidad semipresencial (blended learning) y del uso básico de las TIC, encontraron una aprobación de la plataforma Blackboard, reconociendo al mismo tiempo, la importancia de continuar trabajando con la tecnología y materiales digitales, dado que facilitan la práctica pedagógica.

El Factor Alfabetización Tecnológica consiste en adquirir conocimientos y conceptos acerca de las tecnologías digitales y reconocer los elementos del contexto laboral cuando se usa un ordenador con sus respectivas aplicaciones (Ocampo et al., 2015). Asimismo, la alfabetización digital alude a la capacidad de entender y emplear las fuentes de información transmitida por medio de un ordenador; es decir, se refiere a acciones formativas encaminadas a desarrollar destrezas técnicas, sociales, culturales y educativas (Cabero et al., 2011).

Mayes y Fowler (como se citó en Lankshear \& Knobel, 2008) y Gisbert et al. (2011) coinciden en afirmar que ser alfabetizado tecnológicamente es determinado por el contexto social y cultural donde se desenvuelve cada persona dado que pertenecemos a una sociedad digital. Por lo que se le considera como un rasgo de identidad esencial del ser humano de hoy. Asimismo, ser alfabetizado tecnológicamente supone saber utilizar los ordenadores y los programas más estandarizados para adquirir buenos hábitos de trabajo (Fernández et al., 2019). No obstante, Román-García et al. (2016) consideran que una de las principales dificultades de la alfabetización tecnológica es el constante cambio de la información y aplicaciones tecnológicas; por ello recomiendan estar preparados para identificar y seleccionar la tecnología necesaria para el desarrollo profesional docente de manera continua. 
Los resultados respecto del Factor de Acceso y Uso de la Información indican que los docentes reconocieron la importancia de la digitalización en la transformación de la sociedad en la medida que generan nuevos retos y oportunidades para desenvolverse y solucionar problemas; se requiere que el docente tome conciencia de su capacidad para precisar problemas que resolverá usando las herramientas tecnológicas; identifique los recursos TIC apropiados para buscar información que contribuyan a solucionar problemas; recupere, organice y gestione información con recursos tecnológicos; identifique la información selecta valorando las diferentes fuentes y su origen; sintetice y organice la información selecta para construir y asimilar el reciente conocimiento y demuestre el beneficio del conocimiento alcanzado para tomar decisiones a fin de solucionar un problema.

El acceso y uso de la información es definido como la búsqueda de información selecta evaluando las diferentes fuentes, así como su procedencia. Consiste en la planificación, identificación, discriminación, clasificación, comunicación y organización de la información, respetando la autoría correspondiente (Jaramillo et al., 2011). Además, este factor implica ser autónomo, eficiente, tener la capacidad de criticar y reflexionar sobre la información y herramientas tecnológicas que se pretende seleccionar, tratar y usar; así mismo, actuar crítica y reflexivamente para distinguir la información relevante de la irrelevante (Viñals \& Cuenca, 2016).

Chávez et al. (2016) enfatizan que acceder a, y usar, información constituye un factor esencial para un pertinente desenvolvimiento en la sociedad actual. Utilizar las herramientas tecnológicas supone buscar, obtener, identificar, resumir, organizar y evaluar información para responder de forma más oportuna a las demandas de la sociedad.

Respecto del Factor Comunicación y Colaboración, los docentes calificaron como importante o muy importante utilizar las herramientas y entornos digitales para una eficiente comunicación, interacción y colaboración, así como laborar en procesos, faenas y metas compartidas. El uso de medios digitales permite la producción de documentos colaborativos de manera asíncrona o sincrónicamente, de manera eficaz y fructífera con sus compañeros.

Para Ferrari (2013) e Instituto Nacional de Tecnologías Educativas y Formación del Profesorado [INTEF] (2017) este factor es considerado como la capacidad docente para participar e interactuar en entornos digitales y compartir recursos. Al respecto, Cantón-Mayo et al. (2017) señalan que los docentes deben ser competentes para interactuar mediante los medios digitales, adaptándose a las distintas formas, formatos, estrategias y modos de comunicarse; compartir contenidos, recursos educativos y conocimientos; colaborar y trabajar en equipos en la creación de recursos y contenidos usando medios digitales; conocer y valorar las normas de interacción en línea, teniendo en cuenta la variedad cultural, buscando protegerse de probables riesgos online mediante estrategias activas que detecten actuaciones inapropiadas $\mathrm{y}$, finalmente, gestionar una identidad digital salvaguardando la reputación digital mediante una buena gestión de los datos proporcionados en las diferentes cuentas y aplicaciones que se han utilizado.

En cuanto al Factor de Ciudadanía Digital, se halló un elevado porcentaje en la valoración "muy importante" respecto de asumir un compromiso ético en la utilización legal, segura y responsable de las TIC y de la información digital. Los docentes manifestaron también un sentido de responsabilidad personal para aprender durante toda la vida. Asimismo, para ellos es muy importante fomentar la ciudadanía digital entre colegas, dado que actualmente es prioritario su desarrollo en las instituciones educativas, sobre todo en aquellas que buscan integrar las TIC en el proceso enseñanza aprendizaje, con el objetivo de formar personas para desenvolverse en una sociedad eminentemente digital.

Se entiende por ciudadanía digital, una serie de conocimientos, destrezas y actitudes esenciales para utilizar las tecnologías de forma responsable, instruida, inequívoca, ética, autónoma e interactiva, que haga posible un favorable desenvolvimiento en la sociedad digital en la vivimos hoy, mediante un uso responsable de la huella digital suscitado a partir del ejercicio y examen de los derechos digitales que se poseen (Ministerio de Educación de Chile, 2019). La ciudadanía digital también es definida como aquellas normas de conducta establecidas para prevenir problemas asociados con el inadecuado empleo y abuso de las herramientas tecnológicas (Ribble et al., 2004). La ciudadanía digital ayuda a definir el comportamiento deseable, determinando límites que permitan distinguir cuándo no estamos realizando un uso correcto de la tecnología.

Por último, se evidenciaron las valoraciones de los docentes sobre la Creatividad e Innovación, 
reconociendo en todos los ítems de este factor que es importante o muy importante exponer la incorporación de los conocimientos tecnológicos en el quehacer pedagógico. En relación con ello, Arias et al. (2013), Díaz (2006), Iriarte (2011) y Jiménez (2019) coinciden en manifestar que este factor permite transformar el contexto y generar nuevas ideas para aplicarlas con éxito en el ámbito donde los docentes se desenvuelven. Pero, para ello, es necesario involucrar a las herramientas tecnológicas dentro de las actividades pedagógicas (Jiménez, 2019; Montero, 2009; Villa \& Poblete, 2011; Villalobos, 2009).

En ese sentido, los profesores universitarios tienen la responsabilidad de incorporar en su quehacer pedagógico una serie de conocimientos y estrategias didácticas y tecnológicas que satisfagan los requerimientos sociales. A partir de aquí, se plantea la urgencia del diseño de un perfil de docente universitario con dominio tecnológico. Para alcanzar este dominio se precisa de la adquisición de conocimientos, destrezas y actitudes para aplicar estrategias innovadoras mediadas con el uso de herramientas tecnológicas, a fin de fomentar en los estudiantes un rol activo y mayor compromiso en su PEA.

\section{CONCLUSIONES}

Los docentes universitarios sienten la necesidad de desarrollar sus competencias digitales, a partir de la adquisición de conocimiento de los recursos digitales existentes, así como sobre cómo y dónde encontrarlos e incorporarlos en quehacer diario. En este sentido, las universidades deben incluir en su malla curricular la formación en competencias digitales como una política educativa.

El principal desafío que enfrenta la educación superior es incorporar los recursos tecnológicos al PEA mediante la generalización de los contenidos digitales y la innovación pedagógica. La universidad debe certificar y/o acreditar el dominio y desarrollo de las herramientas tecnológicas de sus docentes en las asignaturas generadas autónomamente y como parte de la colaboración profesional que fomenta la institución.

\section{Correspondencia:}

Jamine Pozú-Franco

Correo electrónico: jamine.pozu.f@upch.pe

\section{REFERENCIAS}

Ander-Egg, E. (2005). Debates y propuestas sobre la problemática educativa. Algunas reflexiones sobre los retos del futuro inmediato. Homo Sapiens.

Arias, C., Giraldo, D., \& Anaya, L. (2013). Competencia creatividad e innovación: conceptualización y abordaje en la educación. Katharsis, 15(1), 195-213. https://doi.org/10.25057/25005731.245

Cabero, J., Llorente, M. C., \& Marín., V. (2011). Las prácticas con TIC: el acercamiento a la Sociedad del conocimiento. ¿Están los estudiantes capacitados? En R. Roig \& C. Laneve (Eds.), La práctica educativa en la Sociedad de la Información. Innovación a través de la Investigación (pp. 71-81). Marfil.

Cantón-Mayo, I., Cañón-Rodríguez, R., \& Grande-dePrado, M. (2017). La comunicación como subdimensión de la competencia digital en futuros maestros de primaria. Revista de Medios y Educación, 50(1), 33-47. http://dx.doi.org/10.12795/pixelbit. 2017.i50.02

Chávez, F. H., Cantú, H., \& Rodríguez, C. M. (2016). Competencias digitales y tratamiento de información desde la mirada infantil. REDIE, 18(1), 209-220. http://www.scielo.org.mx/scielo.php?script $=$ sci arttext\&pid=S1607-40412016000100015

Comisión Europea (2008). Cedefop-Centro Europeopara el Desarrollo de la Formación Profesional. Revista Europea de Formación Profesional, 2(44), 4-9. https:// www.oei.es/historico/pdf2/revista_cedefop_44_ es.pdf

Comunidades Europeas (2007). Competencias clave para el aprendizaje permanente. Un Marco de Referencia Europeo. http://www.mecd.gob.es/ $\mathrm{dctm} / \mathrm{ministerio/educacion/mecu/movilidad-europa/}$ competenciasclave.pdf?documentId $=0901 \mathrm{e} 72$ b80685fb 1

Consejo y Parlamento Europeo (2006). Recomendación del Parlamento Europeo y del consejo de 18 de diciembre de 2006 sobre las competencias clave para el aprendizaje permanente. Diario Oficial de la UnionEuropea.https://eur-lex.europa.eu/LexUriServ/ LexUriServ.do?uri=OJ:L:2006:394:0010:0018: ES:PDF

Díaz, F. (2006). Enseñanza situada. Vínculo entre la escuela y la vida. Mc-Graw Hill Interamericana.

Fernández, E., Ordóñez, E., Morales, B., \& López, J. (2019). La competencia digital en la docencia universitaria. Octaedro.

Ferrari, A. (2012). Digital Competence in Practice: An Analysis of Frameworks. Technical Report, European Commission, Joint Research Centre. https://doi. org $/ 10.2791 / 82116$

Ferrari, A. (2013). DIGCOMP: A framework for developing and understanding digital competence in Europe. Publications Office of the European 
Union. https://doi.org/10.2788/52966

Flores-Lueg, C., \& Roig. R. (2016). Diseño y validación de una escala de autoevaluación de competencias digitales para estudiantes de pedagogía. Pixel-Bit. Revista de Medios y Educación, 48(1), 209-224. http://dx.doi.org/10.12795/pixelbit.2016.i48.14

Gisbert, M., Espuny, C., \& González, J. (2011). Cómo trabajar la competencia digital en estudiantes universitarios. En R. Roig \& C. Laneve (Eds.), La práctica educativa en la Sociedad de la Información. Innovación a través de la Investigación (pp. 157174). Marfil.

Instefjord, E. (2014). Appropriation of digital competence in teacher education. Nordic Journal of Digital Literacy, 9(4), 313-329.

Instituto Nacional de Tecnologías Educativas y Formación del Profesorado (2017). Marco común de competencia digital docente. Ministerio de Educación, Cultura y Deporte. http://educalab.es/documents/10180/12809/ MarcoComunCompeDigiDoceV2.pdf

Iriarte, A. J. (2011). Desarrollo de la competencia resolución de problemas desde una didáctica con enfoque metacognitivo. Zona Próxima, 1(15), 2-21. http://rcientificas.uninorte.edu.co/index.php/zona/ article/viewFile/1171/2355

Jaramillo, P., Hennig, C., \& Rincón, Y. (2011). ¿Cómo manejan información los estudiantes de educación superior?: El caso de la Universidad de La Sabana, Colombia. Información, Cultura y Sociedad, 25(1), 117-143. http://eprints.relis. org $/ 17273$

Jiménez, Y. I. (2019). ¿Cómo desarrollar competencias de creatividad e innovación en la educación superior? Caso: carreras de ingeniería del Instituto Politécnico Nacional. RIDE, 8(18). http://doir.org/10.2393/ride. v9i18.427

Lankshear, C., \& Knobel, M. (2008). Digital literacies: Concepts, policies and practices. Peter Lang.

Marciales, G. P., Barbosa, J. W., \& Castañeda, H. (2015). Development of information skills in college: approaches, models and intervention strategies. Investigación Bibliotecológica: Archivonomía, Bibliotecología e Información, 29(65), 39-72. https:// doi.org/10.1016/j.ibbai.2016.02.014

Mengual, S. (2011). Cuestionario de Competencias Digitales en Educación Superior. EDUTIC. http:// www.edutic.ua.es/cdes

Mengual, S., \& Roig, R. (2012). La enseñanza y las competencias TIC en el contexto universitario. En Y. Sandoval, A. Arenas, E. López, J. Cabero \& J. I. Aguaded (Coords.), Las tecnologías de la información en el contexto educativo: Nuevos escenarios de aprendizaje (pp. 17-32). Universidad SantiagodeCali.https://www.researchgate.net/profile/ Santiago_Mengual-Andres/publication/265509034 las-tecnologias-de-la-informacion_17_32-CAP1/ links/54107fb20cf2f2b29a410e5e.pdf

Ministerio de Educación de Chile (2019). Ciudadanía digital. MINEDUC. https://formacionciudadana. mineduc.cl/wp-content/uploads/sites/46/2019/06/ CiudadaniaDigital-Cuadernillo.pdf

Ministerio de Educación del Perú (2016). El Currículo Nacional de la Educación Básica. MINEDU. http://www.minedu.gob.pe/curriculo/pdf/ curriculo-nacional-2016.pdf

Montero, A. (2009). Las competencias en la educación. Competencias educativas, diseño y desarrollo del currículo en los centros. Guadalturia.

Montero, J.A., Merino, F.J., Monte, A. E. Ávila, J. F., \& Cepeda, M. (2019). Competencias digitales clave de los profesionales sanitarios. Educación Médica, 21(5), 338-344. https://doi.org/10.1016/j.edumed.2019. 02.010

Ocampo, A., Gómez, M. G., \& Zambrano, D. (2015). Percepción del profesor sobre el uso del b-learning para fortalecer competencias laborales. Apertura, 7(2). http://www.udgvirtual.udg.mx/apertura/index. php/apertura/article/view/669/496

Organización para la Cooperación y Desarrollo Económicos (2008). Panorama de la Educación 2008: Nota informativa de la OCDE para México. Organización para la Cooperación y Desarrollo Económicos. http://www.oecd.org/centrodemexico/ medios/41288187.pdf

Ribble, M., S., Bailey, G. D., \& Ross. T. W. (2004). Digital Citizenship, addressing appropriate technology behavior. Learning \& Leading with Technology, 32(1), 6-12. https://files.eric.ed.gov/fulltext/ EJ695788.pdf

Román-García, S., Almansa-Martínez, A., \& Cruz-Díaz, D. M. (2016). Adultos y mayores frente a las TIC. La competencia mediática de los inmigrantes digitales. Comunicar, 24(49), 101-109. http://dx.doi. org/10.3916/C49-2016-10

Tobón, S. (2016). Formación basada en Competencias. Universidad Complutense de Madrid. https://www. uv.mx/psicologia/files/2015/07/Tobon-S.Formacion-basada-en-competencias.pdf

Unión Europea (2011). Competencias clave para el aprendizajepermanente.UniónEuropea.http://eur-lex. europa.eu/legal-content/ES/TXT/? uri= URISERV\%3Ac11090

Villa, A., \& Poblete, M. (2011). Aprendizaje basado en competencias. Una propuesta para la evaluación de las competencias genéricas. Ediciones Mensajero.

Villalobos, M. (2009). Evaluación del aprendizaje basado en competencias. Minos Tercer Milenio.

Viñals, A., \& Cuenca, J. (2016). El rol del docente en la era digital. Revista Interuniversitaria de Formación del Profesorado, 30(2), 103-114.

Zabalza, M. A. (2007). Competencias docentes del profesorado universitario. Calidad y desarrollo profesional. Narcea. 